\title{
Maximal sum-free sets in cyclic groups of prime-power order
}

\section{Anne Penfold Street}

\begin{abstract}
A subset $S$ of an additive group $G$ is called a maximal sum-free set in $G$ if $(S+S) \cap S=\emptyset$ and $|S| \geq|T|$ for every sum-free set $T$ in $G$. In this paper, the maximal sum-free sets in cyclic p-groups are characterized to within automorphism.
\end{abstract}

Given an additive group $G$ and non-empty subsets $S, T$ of $G$, let $S+T$ denote the set $\{s+t ; s \in S, t \in T\}, \vec{S}$ the complement of $S$ in $G$ and $|S|$ the cardinality of $S$. We call $S$ a sum-free set in $G$ if $(S+S) \subseteq \bar{S}$. If, in addition, $|S| \geq|T|$ for every sum-free set $T$ in $G$, then we call $S$ a maximal sum-free set in $G$. We denote by $\lambda(G)$ the cardinality of a maximal sum-free set in $G$.

Exact values of $\lambda(G)$ were given by Diananda and Yap [1] for $|G|$ divisible by 3 or by at least one prime $q \equiv 2$ (3). When every prime divisor of $|G|$ is a prime $p \equiv I$ (3) then, by [1], $|G|(m-1) / 3 m \leq \lambda(G) \leq(|G|-1) / 3$, where $m$ is the exponent of $G$, and it is conjectured that in fact

$$
|G|(m-1) / 3 m=\lambda(G) .
$$

This conjecture was verified in [1] for $z_{n}$, the cyclic group of order $n$, and by Rhemtulla and Street [4] for elementary abelian p-groups.

Maximal sum-free sets have been characterized (up to automorphism) for the following classes of abelian p-groups:

Received 18 December 1970. 
(i) for $z_{p}$, with $p \equiv 2$ (3) in [1] and [6], and with $p \equiv 1$ (3) in [4], (see also partial results in [7]);

(ii) for elementary abelian p-groups, with $p \equiv 2$ (3) in [1], and with $p \equiv 1$ (3) in [5];

(iii) for $z_{p^{\alpha}}$, with $p \equiv 2$ (3) in [1].

Here we extend the argument of [4] to characterize the maximal sum-free sets in $z_{p^{\alpha}}$ with $p \equiv I$ (3). More precisely, we prove the following:

THEOREM. Let $G=z_{p}^{\alpha}$, where $p=3 k+1$ is prime and $p^{\alpha}=3 k_{\alpha}+I$. Then any maximal sum-free set $s$ may be mopped, under some automorphism of $G$, to one of the following:

$$
\begin{aligned}
& A_{\alpha}=\left\{k_{\alpha}, k_{\alpha}+2, \ldots, 2 k_{\alpha}-1,2 k_{\alpha}+1\right\} ; \\
& B_{\alpha}=\left\{k_{\alpha}, \ldots, 2 k_{\alpha}-1\right\} ; \\
& C_{\alpha}=\left\{k_{\alpha}+1, \ldots, 2 k_{\alpha}\right\} .
\end{aligned}
$$

DEFINITION. Let $C$ be a subset and $H$ a subgroup of an abelian group $G$.

(i) $C$ is said to be in arithmetic progression if $C=\{g+i d|i=0,1, \ldots| C \mid-1$,$\} , for some g, d \in G$, $d \neq 0$. If so, $d$ is called a difference of $C$.

(ii) $C$ is said to be aperiodic if $C+H=C$ implies $H=\{0\}$.

(iii) $C$ is said to be periodic if $C+H=C$ for some $H \neq\{0\}$. If so, $H$ is called a period of $C$.

(iv) $C$ is said to be quasiperiodic if $C=C^{\prime} \cup C^{\prime \prime}$, where $C^{\prime} \cap C^{\prime \prime}=\varnothing, C^{\prime}+H=C^{\prime}$ for some $H \neq\{0\}$ and $C^{\prime \prime}$ is contained in one coset of $H$. If so, $H$ is called a quasiperiod of $C$.

Notation. Let $G=2 p^{\alpha}$ and let $H \neq\{0\}$ be a subgroup of 
$G, H=Z_{p^{\beta}}$. If $S$ is a maximal sum-free set in $G$, then $S_{i}$ denotes the subset of $H$ such that $S_{i}+i=S \cap(H+i)$, where $H+1$ generates $G / H$ and $i=0,1, \ldots, p^{\alpha-\beta}-1$.

The proof of the theorem depends primarily on results of Kemperman [2], especially on Theorems 2.1 and 3.4 and lemma 4.3. We also need Kneser's Theorem [3], the lemma of [4] and the following simple results.

LEMMA 1. Let $G=2_{p^{\alpha}}, p=3 k+1$, and let $C$ be a subset of $G$ in arithmetic progression, with difference $d$. If $|C|>p^{\alpha} / 7$, then $d$ has order $p^{\alpha}$.

LEMMA 2. Let $G=Z_{p^{\alpha}}$ where $p=3 k+1$ and $p^{\alpha}=3 k_{\alpha}+1$. Let $H \neq\{0\}$ be a subgroup of $G, H=Z_{p^{\beta}}$, and let $S$ be a maximal sum-free set in $G$.

(i) Let $I=\left\{i\left|i=0,1, \ldots, p^{\alpha-\beta}-1 ;\right| S_{i} \mid \geq\left(p^{\beta}+1\right) / 2\right\}$. Let $L=\left\{\tau\left|\tau=0,1, \ldots, p^{\alpha-\beta}-1 ; S_{\tau}=\phi\right\rangle\right\}$. Then $I+I \subseteq L$.

(ii) If $s_{0} \neq \emptyset$, then $s_{i} \neq H$ for ariy $i=0,1, \ldots, p^{\alpha-\beta}-1$.

(iii) $\lambda(G)>\lambda(G / H)|H|$.

(iv) Suppose the theorem is true for $z_{p}$, for all $\delta<\alpha$. Then $s_{i}=H$ for fewer than $k_{\alpha-\beta}$ values of $i$.

Proof. (i) Since $S$ is sum-free,

$$
\left(S_{i}+S_{j}\right) \cap S_{i+j}=\varnothing \text {. }
$$

By Kneser's Theorem [3], there exists some subgroup $K<H, \quad|K|=p^{\gamma}$, such that $S_{i}+S_{j}+K=S_{i}+S_{j}$ and $\left|S_{i}+S_{j}\right| \geq\left|S_{i}+K\right|+\left|S_{j}+K\right|-|K|$. Since $\left|S_{i}\right| \geq\left(p^{B}+1\right) / 2$, we must have $\left|S_{i}+K\right| \geq\left(p^{\beta}+p^{\gamma}\right) / 2$ and 
similarly for $S_{j}$. Hence $\left|S_{i}+S_{j}\right| \geq 2\left(p^{\beta}+p^{\gamma}\right) / 2-p^{\gamma}=p^{\beta}$ and $S_{i+j}=\varnothing$.

(ii) Apply (2) in the particular case $j=0$.

(iii)

$$
\begin{gathered}
\lambda(G)=k_{\alpha}=k\left(p^{\alpha-1}+\ldots+p+1\right), \\
\lambda(G / H)|H|=k_{\alpha-\beta} p^{\beta}=k\left(p^{\alpha-1}+\ldots+p^{\beta}\right) .
\end{gathered}
$$

(iv) By (i), if $S_{i}=H$ then $i \in I$ which is a sum-free set in $G / H=z_{p^{\alpha-\beta}}$. Hence $S_{i}=H$ for at most $k_{\alpha-\beta}$ values of $i$.

Suppose $S_{i}=H$ for $k_{\alpha-\beta}$ values of $i$ and let $T=\left\{i \in z_{p^{\alpha-\beta}} \mid S_{i}=H\right\}$. Then $T$ may be mapped (under automorphism of $G / H)$ tc one of the sets $A_{\alpha-\beta}, B_{\alpha-\beta}, C_{\alpha-\beta}$.

Now $A_{\alpha-\beta}+A_{\alpha-\beta}=\overline{A_{\alpha-\beta}}$, so if $T=A_{\alpha-\beta}$ then, by (2), $S_{i}=\emptyset$ for all $i \notin T$.

$$
\left(B_{\alpha-\beta}+B_{\alpha-\beta}\right) \cup B_{\alpha-\beta}=\left\{\overline{k_{\alpha}^{-2,} k_{\alpha}^{-1}}\right\} .
$$

Hence if $T=B_{\alpha-\beta}$ then, by (2), $S_{i}=\emptyset$ for all $i \notin T$ except possibly for $i=k_{\alpha}-2$ or $k_{\alpha}-1$. If $k_{\alpha}=2$, then $s_{k_{\alpha}-2}=S_{0}=\phi$ by (ii); if $k_{\alpha}>2$, then $2\left(k_{\alpha}-2\right) \in T$ so, by (2), $s_{k_{\alpha}-2}=\emptyset$. Also $2\left(k_{\alpha}-1\right) \in T$ so $S_{k_{\alpha}-1}=\emptyset$. Hence $S_{i}=\emptyset$ for all $i \notin T$.

A similar argument shows that if $T=C_{\alpha-\beta}$, then $S_{i}=\emptyset$ for all $i \notin T$.

Hence $\lambda(G)=\lambda(G / H)|H|$ which contradicts (iii).

Proof of the Theorem. We proceed by induction on $\alpha$. For $\alpha=1$, the theorem reduces to Theorem 2 of [4].

By [1], for any $\alpha,|S|=k_{\alpha}=\left(p^{\alpha}-1\right) / 3$. Since $S$ is sum-free, we must have $|S+S| \leq 2|S|+1$ and $|S-S| \leq 2|S|+1$. 
Suppose that $|S+S|<2|S|-1$. By Kneser's Theorem [3], there exists a subgroup $H<G, H \neq\{0\}$, such that $S+S+H=S+S$ and $|S+S| \geq 2|S+H|-|H|$. By Lemma 1 of [1], $S+H=S$, which implies that $|H||S|$. Since $|H|=p^{\beta}, \quad 1 \leq \beta<\alpha$, we have a contradiction. Hence $|S+S| \geq 2|S|-1$ and a similar argument shows that $|S-S| \geq 2|S|-1$.

Now $S-S=-(S-S)$ and $0 \in S-S$. Hence $|S-S|$ is odd and can take one of two values: $|S-S|=2|S| \pm 1$.

I. If $|S-S|=2|S|-1$ then, by Theorem 2.1 of [2], either $S-S$ is in arithmetic progression or $S-S$ is quasiperiodic.

Suppose that $S-S$ is quasiperiodic. Now $|S-S|=2 k\left(p^{\alpha-1}+\ldots+p+1\right)-1$. Hence there exists a subgroup $H<G$, $|H|=p^{\beta} \geq p$, such that $S-S$ consists of the union of $2 k\left(p^{\alpha-\beta-1}+\ldots+p+1\right)$ complete cosets of $H$, together with $2 k\left(p^{\beta-1}+\ldots+p+1\right)-1$ elements, all contained in one other coset of $H$. Since $|G / H|=p^{\alpha-\beta}$ and since $S-S=-(S-S)$, these $2 k\left(p^{\beta-1}+\ldots+p+1\right)-1$ leftover elements must belong to $H$ itself. Since $|(S-S) \cup S|=|G|-2$, at least $k\left(p^{\beta-1}+\ldots+p+1\right)$ of the remaining elements of $H$ must belong to $S$. But $k\left(p^{\beta-1}+\ldots+p+1\right)=k_{\beta}=\lambda(H)$, so $\left|s_{0}\right|=k_{\beta}$. So the remaining $k\left(p^{\alpha-\beta-1}+\ldots+p+1\right)$ cosets of $H$ must be contained in $S$, contradicting Lemma 2 (ii).

Hence $S-S$ is in arithmetic progression. Since $|S-S|=2 k_{\alpha}-1$, Lemma $I$ shows that the difference, $d$, of $S-S$ must be of order $p^{\alpha}$. By Lemma 4.3 of [2], $S$ (and $-S$ ) must also be in arithmetic progression with difference $d$. Hence $S$ may be mapped (by some automorphism of $G$ ) to $B_{\alpha}$ or $C_{\alpha}$.

II. If $|S-S|=2|S|+1$, then $S-S=\bar{S}$. Hence $S=-S$, $S+S=S-S$ and we may apply the Lemma of [4].

(a) Suppose that, for some $g \in G,|(S+g) \cap S|=1$. Then by the 
lemma, $|(s+3 g / 2) n s| \geq k_{\alpha}-3$.

If $p \nmid g$, map $3 g / 2$ to $I$ so that $g=k_{\alpha}+1$. The first part of the argument of Theorem 2 of [4] shows that $S$ may. be mapped, under automorphism of $G$, to $A_{\alpha}$.

$$
\begin{gathered}
\text { If } p^{\alpha-\beta} \mid g, p^{\alpha-\beta+1} \not g \text {, map } 3 g / 2 \text { to } p^{\alpha-\beta} \text { so that } \\
g=\left(k p^{\beta-1}+k p^{\beta-2}+\ldots+k p+k+1\right) p^{\alpha-\beta}=\left(k_{\beta}+1\right) p^{\alpha-\beta} \text {. Now } \\
\left|\left(S+p^{\alpha-\beta}\right) n S\right| \geq k_{\alpha}-3 \text {. Let } \quad H=z_{p^{\beta}}=\left\langle p^{\alpha-\beta}\right\rangle \text {. Then } \\
S=p_{i=0}^{u^{\alpha-\beta}}\left(S_{i}+i\right) \text { and }\left|\left(S+p^{\alpha-\beta}\right) n S\right|=\sum_{i=0}^{\alpha-\beta}\left|\left(S_{i}+p^{\alpha-\beta}\right) n S_{i}\right| .
\end{gathered}
$$

Note that $S_{i}+p^{\alpha-\beta}=S_{i}$ if and only if $S_{i}=\emptyset$ or $S_{i}=H$.

If $\left|\left(S+p^{\alpha-\beta}\right) \Omega S\right|=k_{\alpha}$, then $S$ consists of a union of complete cosets of $H$. Hence $|H||| S \mid$ which is a contradiction.

If $k_{\alpha}-1 \geq\left|\left(S+p^{\alpha-\beta}\right) n S\right| \geq k_{\alpha}-3$, we have to consider several possibilities for $S$ :

(i) $S$ consists of a union of $k\left(p^{\alpha-\beta-1}+\ldots+p+1\right)=k_{\alpha-\beta}$ complete cosets of $H$, together with $k\left(p^{\beta-1}+\ldots+p+1\right)$ other elements distributed between one, two or three other cosets of $H$. But this contradicts Lemma 2 (iv).

(ii) $S$ consists of a union of $k_{\alpha-\beta}-1$ complete cosets of $H$, together with $p^{\beta}+k\left(p^{\beta-1}+\ldots+p+1\right)$ other elements distributed between two or three other cosets of $H$. But since $S=-S$, one of the complete cosets must be $H$ itself, contradicting the sum-freeness of $S$.

(iii) $S$ consists of a union of $k_{\alpha-\beta}-2$ complete cosets of $H$, together with $2 p^{\beta}+k\left(p^{\beta-1}+\ldots+p+1\right)$ other elements distributed 
between three other cosets of $H$. Since $S=-S$, one of these three other cosets must be $H$ itself. Since $\lambda(H)=k_{B}=k\left(p^{\beta-1}+\ldots+p+1\right)$, at most $k_{\beta}$ of the remaining elements belong to $H$, and in fact $k_{\alpha-\beta}$ complete cosets of $H$ are contained in $S$. This is impossible by (i).

(b) We are now left with the case where $|(S+g) n S| \neq 1$ for any $g \in G$.

(i) Suppose that by taking an automorphism of $G$, we may ensure that $|(S+1) n S| \geq|(S+g) n S|$ for all $g \in G$. We list the elements of $S$ as follows :

$$
S=\left\{a_{1}, \ldots, a_{1}+z_{1}, a_{2}, \ldots, a_{2}+z_{2}, \ldots, a_{h}, \ldots, a_{h}+z_{h}\right\}
$$

where $0<a_{1} \leq a_{1}+z_{1}<a_{2}-1<a_{2}+z_{2}<\ldots<a_{h}-1<a_{h}+z_{h}<p^{\alpha}$ and $a_{i}, \ldots, a_{i}+z_{i}$ denotes a string of $\left(z_{i}+1\right)$ consecutive elements of $s$. Since $S=-S$,

$$
a_{h-i}+z_{h-i}=p^{\alpha}-a_{i+1} \text {, for all } i=0,1, \ldots, h-1 \text {, }
$$

and $|(S+1) n S|=k_{\alpha}-h \geq|(S+g) n S|$ for all $g \in G$. Hence $h$ is minimal in (3) and we show that $h=2$.

Let $X=\left\{a_{1}, \ldots, a_{h}\right\}$ and let

$$
y=\left\{a_{1}+z_{1}+1, \ldots, a_{h}+z_{h}+1\right\}=\left\{1-a_{1}, \ldots, 1-a_{h}\right\}=1-X
$$

by (4). A repetition of the argument of [4] shows that $\left|\left(S+a_{i}-1\right) n S\right| \geq h-1$ and

$$
h \geq\left|\left(X+a_{i}\right) \cap Y\right| \geq h-1 \text { for all } i=1, \ldots, h .
$$

If $|X+X| \geq 2 h-1$, the argument of [4] shows that $h=2$ and $S$ maps under automorphism to $A_{\alpha}$.

If $|X+X| \leq 2 h-2$, then by Kneser's Theorem [3], $X+X$ is periodic so that for some subgroup $H<G, H=Z_{p^{\beta}}$, we have $X+X+H=X+X$ and $|X+X| \geq 2|X+H|-|H|$. Using Theorem 3.4 of [2], we can construct all 
possible sets $X$. We choose a subset $X^{*}$ of $G / H$ such that $X^{*}+X^{*}$ is aperiodic in $G / H$ and $\left|X^{*}+X^{*}\right|=2\left|X^{*}\right|-1$. If $\sigma$ denotes the natural mapping of $G$ to $G / H$, then $X$ can be any subset of $\sigma^{-1} X^{*}$, such that $\left|\sigma^{-1} X^{*} \cap \bar{X}\right| \leq\left(p^{\beta}-1\right) / 2$. Hence any coset of $H$ which contains the first element of a string of elements of $S$ must contain the first elements of at least $\left(p^{\beta}+1\right) / 2$ strings of $S$.

By (5), $X+X$ contains all of $Y$ except possibly one element, say $y$. Since $X+X$ consists of a union of complete cosets of $H$, $(H+y) \cap Y=\{y\}$. Since $Y=1-X$, this implies that $\left|\sigma^{-1} X^{*} \cap \bar{X}\right| \geq p^{\beta}-1$ which is impossible. Hence $Y \subseteq X+X$. We can now describe the distribution of the strings of $S$. Suppose

$$
X^{*}=\left\{H+i_{1}, \ldots, H+i_{2}\right\} \text { for some } i_{1}, \ldots, i_{2} \in\left\{0,1, \ldots, p^{\alpha-\beta}-1\right\} .
$$

In each coset $H+i_{j}$, more than half of the elements of the coset are starting points of strings of $S$. Since $S=-S$, the strings finish in the cosets of $-X^{*}$. If a string finishes in $H-i_{j}$, then the next coset $H+\left(1-i_{j}\right) \in Y \subseteq X+X$. Hence no string can continue into this coset, and similarly no string could pass through $H+i_{j}-1$.

Hence any coset which contains an element of $S$ contains at least $\left(p^{\beta}+1\right) / 2$ elements of $S$. By Lemma 2 ( $\left.i\right)$, the cosets containing elements of $S$ must therefore form a sum-free set in $G / H$. Hence $|S|=\lambda(G) \leq \lambda(G / H)|H|$, contradicting Lemma 2 (iii).

(ii) Finally suppose that by taking an automorphism of $G$, we may ensure that $\left|\left(S+p^{\alpha-\beta}\right) \cap S\right| \geq|(S+g) \cap S|$ for all $g \in \bar{S}$ and that $\left|\left(S+p^{\alpha-\beta}\right) n S\right|>|(S+g) n S|$ for all $g \in \bar{S}$ such that $p^{\alpha-\beta} \mid g$. Let $H=\left\langle p^{\alpha-\beta}\right\rangle=2_{p^{\beta}}$ and let $q=p^{\alpha-\beta}$ for the remainder of this section.

For each $i=0,1, \ldots, q-1$, we have $s_{i}=\emptyset$ or $S_{i}=H$ or 


$$
\begin{array}{r}
s_{i}=\left\{a_{1 i} q, \ldots,\left(a_{1 i}+z_{1 i}\right) q, a_{2 i} q, \ldots,\left(a_{2 i}+z_{2 i}\right) q, \ldots\right. \\
\left.\ldots, a_{\nu_{i} i} q, \ldots,\left(a_{\nu_{i} i^{+}} \nu_{\nu_{i} i}\right) q\right\}
\end{array}
$$

where $0<a_{1 i} \leq a_{1 i}+z_{1 i}<a_{2 i}-1<a_{2 i}+z_{2 i}<\ldots<a_{v_{i}-1}<a_{v_{i} i^{+}}<v_{i^{i}}<p^{\beta}$ and $a_{j i} q, \ldots,\left(a_{j i}+\tau_{j i}\right) q$ denotes the set of $\left(z_{j i}+1\right)$ consecutive multiples of $q$ which we call an H-string in $S$.

$$
\text { Let } I=\left\{i|i=0,1, \ldots, q-1 ; 1 \leq| s_{i} \mid \leq p^{B}-1\right\} \text {. Since }
$$

$S_{i}+q=S_{i}$ if and only if $i \notin I$, we have

$$
|(S+q) n S|=|S|-\sum_{i \in I} \nu_{i}>|(S+g) n S| \text { for all } g \in G, q \mid g .
$$

Let $x=\left\{a_{j i} q+i \mid i=0,1, \ldots, q-1 ; j=1, \ldots, \nu_{i}\right\}$. Since $s=-S$,

we have $\nu_{i}=\nu_{q-i}$ and $\left(a_{j i}+z_{j i}\right) q+i=p^{\alpha}-\left[a_{\nu_{i}-j+1, q-1} q+(q-i)\right]$,

implying that

$$
\left(a_{j i}+z_{j i}\right) q=p^{\alpha}-a_{v_{i}-j+1, q-i} q
$$

Let

$$
\begin{aligned}
Y & =\left\{\left(a_{j i}+z_{j i}+1\right) q+i \mid i=0,1, \ldots, q-1 ; j=1, \ldots, v_{i}\right\} \\
& =q-X \text { by }(7) .
\end{aligned}
$$

Now $\left(a_{j i}-1\right) q+i \in \bar{S}$ so, by (6) and the lemma of [4],

$$
\left|\left[S+\left(a_{j i}-1\right) q+1\right] n s\right| \geq\left(\sum_{i \in I} \nu_{i}\right)-1=|X|-1 .
$$

But for any $s_{1}, s_{2} \in S, s_{1}+\left(a_{j i}-1\right) q+i=s_{2}$ implies that $s_{1} \in X$, $s_{2} \in-X$ and $s_{1}+a_{j i} q+i \in Y$. Hence

(8) $\quad|X|=\sum_{i \in I} v_{i} \geq\left|\left(X+a_{j i} q+i\right) \cap Y\right| \geq\left(\sum_{i \in I} v_{i}\right)-1=|X|-1$

for all $j, i$. 
If $|X+X| \geq 2|X|-1$, then $X+X$ contains at least $|X|-1$ elements of $\bar{Y}$ but $X+a_{j i} q+i$ contains at most one element of $\bar{Y}$. Thus for at least $|X|-2$ values of $(j, i)$, we have $2\left(a_{j i} q+i\right) \mid Y$. But $2\left(a_{j i} q+1\right) \notin Y$ implies that $q\left(1-a_{j i}\right)-i \notin X+a_{j i} q+i$, since $Y=q-X$. Hence for at least $|X|-2$ values of $(j, i)$,

$$
\begin{aligned}
\left(X+a_{j i} q+i\right) \cap Y & =\left\{\left(a_{m}+a_{j i}\right) q+i+n \mid a_{m n^{q}}+n \in X,(m, n) \neq(j, i)\right\} \\
& =\left\{q-\left(a_{m} q+n\right) \mid a_{m n} q+n \in X,(m, n) \neq(j, i)\right\} .
\end{aligned}
$$

Hence, summing these two expressions for the elements of $\left(X+a_{j i} q+i\right) \cap Y$, we have

$$
(|x|-3)\left(a_{j i} q+i\right) \equiv(|x|-1) q-2 \sum_{n \in I} \sum_{m=1}^{\nu_{n}}\left(a_{m n} q^{+n}\right) \quad\left(\bmod p^{\alpha}\right) .
$$

Hence $|X| \leq 3$ and $S$ contains at most three H-strings, together with complete cosets of $H$.

$$
\text { If }|X|=0, S \text { is a union of cosets of } H \text {. This implies }|H||| S \mid
$$
which is a contradiction.

If $|x|=1$ or 3 , then $S=-S$ implies $S_{0} \neq \emptyset$. By Lemma 2 (ii), $S_{i} \neq H$ for any $i$. Hence

$$
|S| \leq \lambda(H)+2(|H|-1)=7\left(p^{B}-1\right) / 3=7 k_{B}<\lambda(G)
$$

by Lemma 2 ( $i$ ii), since $p \geq 7$.

$$
\begin{aligned}
& \text { If }|X|=2 \text {, then } S=-S \text { implies that either } \nu_{0}=2 \text { and } S_{0} \neq \emptyset \\
& \text { or } v_{i}=v_{q-i}=1 \text { for some } i .
\end{aligned}
$$

By the previous argument, we must have $S_{0}=\varnothing$. Hence $S$ consists of a union of $2 \lambda$ cosets of $H$ together with two $H$-strings, each of length at most $p^{\beta}-1$.

Thus

$$
|S|=k_{\alpha} \leq 2 \lambda p^{\beta}+2\left(p^{\beta}-1\right)=2(\lambda+1) p^{\beta}-2 \text {. }
$$


Since $S$ is sum-free, $2 \lambda \leq k_{\alpha-\beta}$. If $2 \lambda \leq k_{\alpha-\beta}-2$, then (9) becomes

$$
k_{\alpha} \leq k_{\alpha-\beta} p^{\beta}-2
$$

which is a contradiction by Lema 2 (iii). But if $2 \lambda=k_{\alpha-\beta}$, we have a contradiction by Lemma 2 (iv).

If $|X+X| \leq 2|X|-2$, then by Kneser's Theorem [3], $X+X$ is periodic and for some subgroup $K<G, K=Z_{p}^{\gamma}$, we have $X+X+K=X+X$ and $|X+X| \geq 2|X+K|-|K|$. We now apply the argument of (b) (i) to $G / K$, using (8) instead of (5). If $K \geq H$, then any coset of $K$ is a union of cosets of $H$. By the previous argument, any coset of $K$ which contains an element of $X$ must contain at least $\left(p^{\gamma}+1\right) / 2$ elements of $X$. Hence there exists a coset of $H$, more than half of whose elements are starting-points of $H$-strings in $S$. This is clearly impossible, so $S$ must consist of a union of complete cosets of $H$. But this implies $|H||| S \mid$ which is a contradiction.

If $K<H$, so that any coset of $H$ is a union of cosets of $K$, then the argument of (b) (i) shows that any coset of $K$ which contains any element of an $H$-string in $S$ must contain at least $\left(p^{\gamma}+1\right) / 2$ elements of $H$-strings in $S$. Hence for each coset, $K+i$, of $K$ either $(K+i) \cap S=\varphi$ or $|(K+i) \cap S| \geq\left(p^{\gamma}+1\right) / 2$. But by Lemma 2 ( $\left.i\right)$, the cosets of $K$, more than half of whose elements belong to $S$, form a sum-free set in $G / K$. Hence $|S|=\lambda(G) \leq \lambda(G / K)|K|$, contradicting Lemma 2 (iii).

\section{References}

[1] Palahenedi Hewage Diananda and Hian Poh Yap, "Maximal sum-free sets of elements of finite groups", Proc. Japan Acad. 45 (1969), 1-5.

[2] J.H.B. Kemperman, "On small sumsets in an abelian group", Acta Math. 103 (1960), 63-88. 
[3] Henry B. Mann, Addition theorems: The addition theorems of group theory and number theory (Interscience Tracts in Pure and Applied Mathematics, number 18; John Wiley, New York, London, Sydney, 1965).

[4] A.H. Rhemtulla and Anne Penfold Street, "Maximal sum-free sets in finite abelian groups", Bulz. Austral. Math. Soc. 2 (1970), 289-297.

[5] A.H. Rhemtulla and Anne Penfold Street, "Maximal sum-free sets in elementary abelian p-groups", Canad. Math. Bulz. (to appear).

[6] H.P. Yap, "The number of maximal sum-free sets in $c_{p}$ ", Nanta Math. 2 (1968), 68-71.

[7] H.P. Yap, "Structure of maximal sum-free sets in $C_{p}$ ", Acta Arith. 17 (1970), 29-35.

University of Queensland,

St Lucia,

Queensland. 